\title{
LETTERS
}

\section{FDG-PET in diagnostic work-up of pulmonary artery}

\section{sarcomas}

\section{To the Editors:}

Pulmonary artery sarcomas (PAS) represent a group of rare tumours with a poor prognosis. In a recent review, the mean \pm SD survival of patients with this disease was $11 \pm 3$ months when no curative resection was attempted, whereas patients undergoing curative resection had a survival of 36.5 \pm 20.2 months [1]. Appropriate application and interpretation of different imaging modalities facilitates differentiation of PAS from chronic thromboembolism. Findings in chest computed tomography (CT) could be inhomogeneous attenuation, vascular distension or filling defects of the entire luminal diameter of a proximal or main pulmonary artery [2, 3]. Extraluminal extension of the tumour can be a specific finding of PAS. Magnetic resonance imaging (MRI) aids the determination of the extent of masses and the relationship to adjacent structures, and during postoperative follow-up [4]. The degree of contrast enhancement in MRI was shown to correlate with the degree of tumour differentiation, and contrast enhancement might be used to differentiate neoplasm from central pulmonary embolism [5]. Single case reports have shown that fluorodeoxyglucose-positron emission tomography (FDG-PET) might be helpful in differentiating PAS from pulmonary thromboembolic disease, and helps decision-making when it is not possible to perform intraoperative biopsies for histological
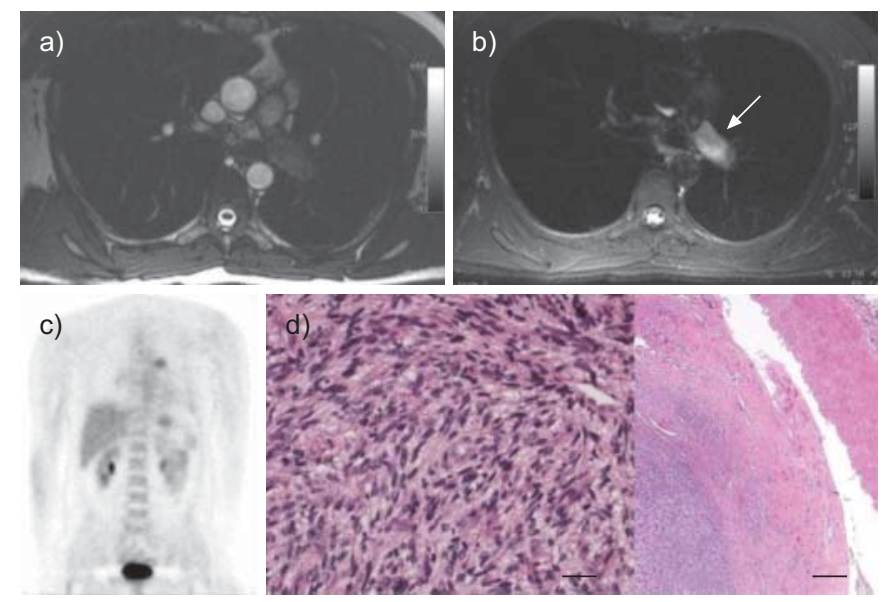

FIGURE 1. Thoracic magnetic resonance imaging (MRI), fluorodeoxyglucosepositron emission tomography (FDG-PET) scan and histological specimen of the excised mass of the first patient. The MRI shows a) an intraluminal mass in the left main pulmonary artery in the True-FISP sequence, with b) high signal intensity on TIRM sequence (arrow). c) The FDG-PET scan shows an increased metabolic activity in the intraluminal mass, and d) histologically confirmed angiosarcoma with a grade 2 in the FNCLCC (Fédération Nationale des Centres de Lutte Contre le Cancer) classification. Scale bars $=50$ and $500 \mu \mathrm{m}$ for the left and right parts of d), respectively. confirmation of PAS before attempting curative pneumonectomy [6, 7]. In the current study, we present three cases of patients with PAS, in whom a presumptive diagnosis of PAS was made preoperatively based on clinical, CT and MRI findings and a positive FDG-PET scan.

Case 1 was a 48-yr-old male nonsmoker with absence of previous medical history who presented with progressive dyspnoea. On spiral contrast-enhanced CT scan taken 4 months prior to presentation, a left-sided paracentral pulmonary embolism was suspected. No improvement occurred with oral anticoagulation. MRI showed an intraluminal mass in the left main pulmonary artery with high signal intensity on T2weighted images (fig. 1a and b). Due to a strong suspicion of a non-thromboembolic process such as neoplasm, an FDG-PET scan was performed, resulting in increased metabolic activity in the intraluminal mass (fig. 1c). The patient subsequently underwent an extended left pneumonectomy with reconstruction of the right outflow tract using a Hancock conduit. Histologically, the arterial intraluminal tumour consisted of spindle cells without vascular structures, with expression of one of three endothelial vascular markers immunhistochemically, and a moderate proliferation fraction, with foci of giant cells and
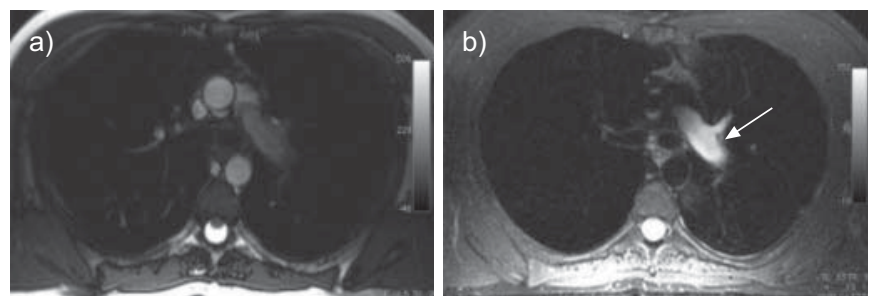

c)
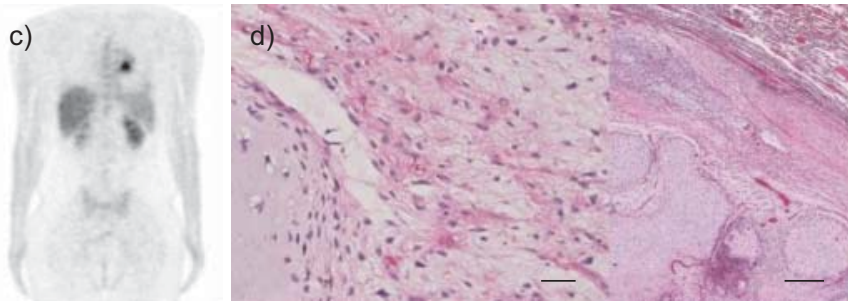

FIGURE 2. Thoracic magnetic resonance imaging (MRI), fluorodeoxyglucosepositron emission tomography (FDG-PET) scan and histological specimen of the excised mass of the second patient. The MRI shows a) an intraluminal mass in the left main pulmonary artery in the True-FISP sequence, with b) high signal intensity on TIRM sequence (arrow). c) The FDG-PET scan shows a focal uptake in the region of the left hilum consistent with active tumour. d) Histological findings equalled an intimal sarcoma with a grade 2 in the FNCLCC (Fédération Nationale des Centres de Lutte Contre le Cancer) classification. Scale bars $=50$ and $500 \mu \mathrm{m}$ for the left and right parts of d), respectively. 

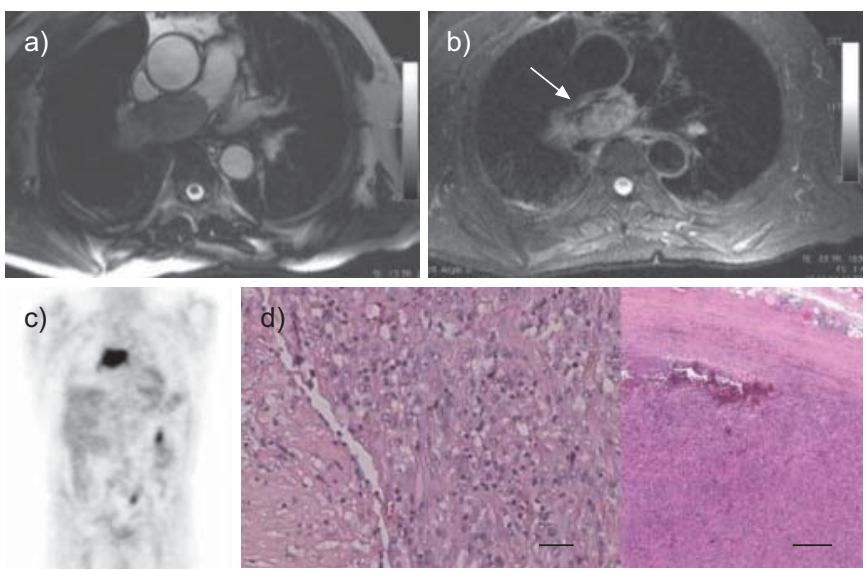

FIGURE 3. Thoracic magnetic resonance imaging (MRI), fluorodeoxyglucosepositron emission tomography (FDG-PET) scan and histological specimen of the excised mass of the third patient. The MRI shows a) a mass in the right pulmonary artery in the True-FISP sequence, with b) high signal intensity on TIRM sequence (arrow). c) The mass showed an increased FDG uptake. d) Histological findings were consistent with an intimal sarcoma with a grade 3 in the FNCLCC (Fédération Nationale des Centres de Lutte Contre le Cancer) classification. Scale bars $=50$ and $500 \mu \mathrm{m}$ for the left and right parts of d), respectively.

foci of primary bone tissue. The findings corresponded to an angiosarcoma with a grade 2 in the FNCLCC (Fédération Nationale des Centres de Lutte Contre le Cancer)-classification (fig. 1d). All lymph nodes and resection margins were free of tumour. The postoperative course was uncomplicated and dyspnoea was markedly improved. There was no sign of tumour relapse 63 months postoperatively.

Case 2 was a 30-yr-old nonsmoking male with a past medical history of pneumonia who presented with chronic progressive dyspnoea and haemoptysis. A spiral CT scan performed 4 months prior to presentation showed a large intravascular filling defect. Anticoagulation remained ineffective. MRI showed a mass in the left pulmonary artery with high signal intensity on T2-weighted images (fig. 2a and 2b). FDG-PET showed a focal uptake (SUV 5.2) in the region of the left hilum consistent with active tumour (fig. 2c). An extended left pneumonectomy with reconstruction of the pulmonary artery using a xeno-pericardial patch was performed. The specimen revealed an intra-arterial spindle cell tumour with chondromatous foci, giant cells, few necrotic areas, focal expression of one of three endothelial vascular markers immunhistochemically, and a moderate proliferation fraction. The histological findings equalled an intimal sarcoma with a grade 2 in the FNCLCC classification (fig. 2d). The postoperative course was uncomplicated and dyspnoea markedly improved. 52 months postoperatively, intrapulmonary and skeletal metastases were diagnosed. The patient underwent re-operation and radiotherapy and was alive 56 months after the first operation.

Case 3 was a 78-yr-old nonsmoking male who presented with chronic progressive dyspnoea and chest pain. On spiral CT scan performed 2 months prior to presentation, a pulmonary embolism was described. Oral anticoagulation was started but did not improve the clinical condition. MRI demonstrated a mass in the right pulmonary artery with high signal intensity on T2-weighted images (fig. $3 a$ and $b$ ). The central lung lesion at the right hilum showed an increased FDG uptake characteristic for malignancy (fig. 3c). The patient underwent extended right pneumonectomy with reconstruction of the pulmonary artery using a xeno-pericardial patch. The histological findings equalled, as in case 2, an intimal sarcoma with a grade 3 in the FNCLCC classification (fig. 3d). All lymph nodes and resection margins were free of tumour. The postoperative course was uncomplicated and dyspnoea markedly improved. There was no sign of tumour relapse 41 months postoperatively.

Pulmonary artery sarcoma should be suspected in cases of large intravascular filling defects without previous acute episode of respiratory or cardiovascular symptoms. In our cases, late contrast-enhancement CT scan that might have indicated a filling defect due to hypervascularised tissue was not available, as it is not performed systematically in patients with suspected pulmonary embolism. The absence of clinical improvement on oral anticoagulation and the unilateral distribution of a massive perfusion defect led to the suspicion of pulmonary artery sarcoma. Dynamic contrast-enhanced MRI underlined this suspicion but, as shown in literature and in our cases, considerable variability of contrast agent uptake exists, possibly reflecting the diverse biological entities and histological pattern of these sarcomas [5]. To confirm the malignant nature of the tumour, FDG-PET was performed and showed in all cases an intensive FDG uptake. As pulmonary thromboembolic disease shows a less intensive FDG uptake, the malignant nature of the filling defect was very likely [8]. In the literature, the utility of FDG-PET scans as a component of the initial staging evaluation in PAS is unclear and needs to be further explored. The sensitivity of PET scanning for soft tissue and bone sarcomas ranges $83-93 \%$ and is greater for high- and intermediate-grade sarcomas [9]. There are only few single case reports that show utility of FDG-PET in the preoperative work-up of patients with suspicion of PAS [6, 7]. In a recent report of a patient who had pulmonary artery embolism due to a thrombus formed on a pulmonary artery leiomyosarcoma, integrated FDG-PET CT led to the correct diagnosis [10]. We are the first to describe a series of patients with PAS in which FDG-PET was an integral part of preoperative evaluation. This approach allowed planning and performance of curative resection, and led to long-term survival of the three patients. We suggest that the preoperative diagnostic work-up in patients with a suspected PAS should include a PET scan to complete the initial staging, which may facilitate intra- and perioperative management and improve outcome.

\section{Tueller*, R. Fischer Biner*, S. Minder*, M. Gugger", C.} Stoupis ${ }^{\uparrow}$, T.M. Krause ${ }^{+}$, T.P. Carrel ${ }^{\S}$, R.A. Schmid ${ }^{f}$, P. Vock and L.P. Nicod*

Depts of *Pulmonary Medicine, "Diagnostic Radiology, ${ }^{+}$Nuclear Medicine, ${ }^{\S}$ Cardiovascular Surgery, ${ }^{f}$ Thoracic Surgery and "Institute for Pathology, University Hospital Berne, Berne, Switzerland.

Correspondence: C. Tueller, Dept of Pulmonary Medicine, Inselspital, 3010 Berne, Switzerland. E-mail: claudia.tueller@ insel.ch

Statement of Interest: None declared. 


\section{REFERENCES}

1 Blackmon SH, Rice DC, Correa AM, et al. Management of primary pulmonary artery sarcomas. Ann Thorac Surg 2009; 87: 977-984.

2 Cox JE, Chiles C, Aquino SL, et al. Pulmonary artery sarcomas: a review of clinical and radiologic features. J Comput Assist Tomogr 1997; 21: 750-755.

3 Kauczor HU, Schwickert HC, Mayer E, et al. Pulmonary artery sarcoma mimicking chronic thromboembolic disease: computed tomography and magnetic resonance imaging findings. Cardiovasc Intervent Radiol 1994; 17: 185-189.

4 Rafal RB, Nichols JN, Markisz JA. Pulmonary artery sarcoma: diagnosis and postoperative follow-up with gadolinium-diethylenetriamine pentaacetic acid-enhanced magnetic resonance imaging. Mayo Clin Proc 1995; 70: 173-176.

5 Kacl GM, Bruder E, Pfammatter T, et al. Primary angiosarcoma of the pulmonary arteries: dynamic contrast-enhanced MRI. J Comput Assist Tomogr 1998; 22: 687-691.
6 Thurer RL, Thorsen A, Parker JA, et al. FDG imaging of a pulmonary artery sarcoma. Ann Thorac Surg 2000; 70: 1414-1415.

7 Chong S, Kim TS, Kim BT, et al. Pulmonary artery sarcoma mimicking pulmonary thromboembolism: integrated FDG PET/ CT. AJR Am J Roentgenol 2007; 188: 1691-1693.

8 Wittram C, Scott JA. ${ }^{18}$ F-FDG PET of pulmonary embolism. AJR Am J Roentgenol 2007; 189: 171-176.

9 Bastiaannet E, Groen H, Jager PL, et al. The value of FDG-PET in the detection, grading and response to therapy of soft tissue and bone sarcomas; a systematic review and meta-analysis. Cancer Treat Rev 2004; 30: 83-101.

10 Farsad M, Pernter P, Triani A, et al. Thromboembolism in pulmonary artery sarcoma. Clin Nucl Med 2009; 34: 239-240.

DOI: 10.1183/09031936.00114708

\section{Glomus vagale presenting as chronic cough}

\section{To the Editors:}

Chronic cough is one of the most common symptoms of medical importance with $12 \%$ of the population reporting significant symptoms on a daily or weekly basis. The differential diagnosis of chronic cough is extensive. Uncommon causes of chronic cough may be missed unless an adequate history is obtained, a detailed examination performed and appropriate investigations arranged. Herein, we present a case of chronic cough, the aetiology of which was previously unreported.

A 57-yr-old female presented with a history of chronic cough for 12 months. The cough had features of reflux [1] as it was precipitated by food and phonation, and associated with frequent clearing of the throat. Moreover, the cough was worse when she was lying flat and started upon rising in the morning. In addition, she described her cough to be precipitated by upward movement of her neck, for example looking at the top shelf in a supermarket. There was no history of decreased appetite or weight loss. She was known to have a hiatus hernia. Her past medical history was otherwise unremarkable. She was a life long nonsmoker and was not on any regular medications. In view of clinical history being highly suggestive of reflux cough, she was started on treatment with lansoprazole in combination with ranitidine; however, she did not respond.

On the second consultation, fullness on the right side of her neck was observed. On examination, a prominent, distended right jugular vein was observed, suggesting the possibility of vascular lesion in the neck. A computed tomography (CT) scan of the neck revealed a brightly enhancing mass of the carotid sheath splaying the internal carotid artery and the internal jugular vein (fig. 1a). Magnetic resonance imaging (MRI) confirmed the CT scan findings of a lobulated and well circumscribed mass measuring $2 \times 2 \times 3.5 \mathrm{~cm}$ in diameter extending from just above the carotid bifurcation to just below the skull base. The lesion enhanced significantly following gadolinium injection. Magnetic resonance angiography (MRA) demonstrated multiple vessels within the lesion (fig. 1b). In addition, there appeared to be redundancy of the right aryepiglottic fold with slight medial displacement of the right vocal cord suggestive of recurrent laryngeal nerve palsy. These radiographic features suggested the mass was a "glomus vagale". Surgical resection of the tumour was successfully undertaken following pre-operative embolisation. The histology from the tumour showed a mixture of trabecular and nested patterns of cells with strongly positive staining to S-100, synaptophysin and chromogranin, consistent with a diagnosis of vagal paraganglioma. Investigations for systemic associations revealed normal urinary catecholamine levels. Postoperatively, she developed a hoarse voice and worsening regurgitation demonstrating expected vagal damage during surgical resection. However, coughing secondary to neck movement completely ceased following the removal of the tumour.
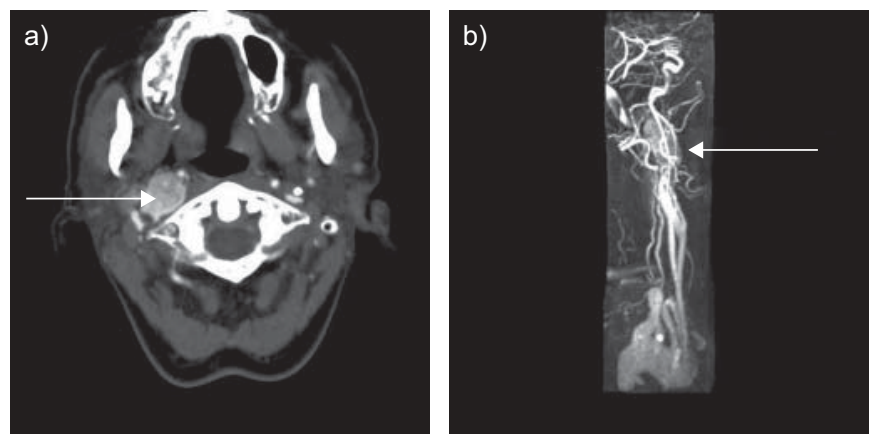

FIGURE 1. a) Contrast-enhanced computed tomography image of the neck showing a brightly enhancing mass (arrow) separating the internal carotid and internal jugular vein on the right side. b) Contrast-enhanced magnetic resonance angiography demonstrating multiple vessels within the mass (arrow) along the right carotid sheath extending from just above the carotid bifurcation to just below the skull base. 